Trauma Berufskrankh 2008 10 [Suppl 2]:196-199

DOI 10.1007/s10039-008-1398-5

Online publiziert: 26. April 2008

(c) Springer Medizin Verlag 2008

\author{
W. Schaffartzik ${ }^{1,2} \cdot$ J. Neu ${ }^{2}$ \\ ${ }^{1}$ Klinik für Anästhesiologie, Intensivmedizin und Schmerztherapie, \\ Unfallkrankenhaus, Berlin \\ ${ }^{2}$ Schlichtungsstelle für Arzthaftpflichtfragen \\ der Norddeutsche Ärztekammern, Hannover
}

\title{
Definition des ärztlichen Behandlungsfehlers
}

$\mathrm{Zu}$ einer Umverteilung der Beweislast hinsichtlich der Kausalität kann es z. B. bei einem schweren Behandlungsfehler kommen. Ein Behandlungsfehler ist als schwer zu beurteilen, wenn der Arzt eindeutig gegen bewährte ärztliche Handlungsregeln oder gesicherte medizinische Erkenntnisse verstoßen und einen Fehler begangen hat, der aus objektiver ärztlicher Sicht nicht mehr verständlich erscheint, weil er einem Arzt schlechterdings nicht unterlaufen darf [11]. Liegt ein derartiger Fehler vor, reicht es für den Kausalitätsnachweis bereits aus, wenn der Fehler zumindest geeignet ist, den eingetretenen Gesundheitsschaden verursacht haben $\mathrm{zu}$ können, es sei denn, die Arztseite könnte beweisen, dass der Gesundheitsschaden völlig unabhängig vom Fehler eingetreten ist.

$\mathrm{Zu}$ Beweislasterleichterungen zugunsten des Patienten kommt es nicht nur bei einem schweren Behandlungsfehler, sondern auch im Zusammenhang mit der Risikoaufklärung, bei Mängeln in der Befunderhebung, Dokumentationsmängeln, in Fällen des Anscheinsbeweises und bei dem als vollbeherrschbar eingestuften Risiko (z. B. Lagerung eines Patienten, Medizintechnik).

\section{Standards in der Medizin}

Ob der bei einem Patienten im Zusammenhang mit der Behandlung eingetretene Gesundheitsschaden die Folge einer als unvermeidbar anzusehenden Komplikation oder die Folge eines Behandlungs- fehlers darstellt, wird über die Betrachtung des zum Zeitpunkt der Behandlung des Patienten gültigen Standards des entsprechenden medizinischen Fachgebiets und der daraus abzuleitenden erforderlichen Sorgfaltspflicht des Arztes entschieden. Dabei ist zu berücksichtigen, dass der Standard in Krankenhäusern, z. B. zwischen einem Allgemeinen Krankenhaus und einer Universitätsklinik, unterschiedlich sein kann, ohne dass aus dieser Situation bereits ein Vorwurf gegen den Arzt zu begründen wäre [9].

Die Feststellung des nicht eingehaltenen Standards kann deswegen problematisch werden, weil derzeit nicht für jede medizinische Behandlung ein Standard definiert ist. Dies wird auch in naher $\mathrm{Zu}$ kunft nicht der Fall sein. Es ist die Aufgabe des medizinischen Sachverständigen, wissenschaftlich begründet festzustellen, ob der Arzt bei der Behandlung so reagiert hat, wie es von einem ordentlichen, pflichtgetreuen Arzt in der konkreten Situation erwartet werden konnte.

Das im Folgenden angeführte Beispiel soll die Problematik der Definition des Standards verdeutlichen.

Beispiel. Ein Patient hatte nach einer trachealen Intubation einen Schaden an der Trachea erlitten. Er warf dem Arzt vor, dass der Druck im Cuff des Tubus nicht gemessen worden sei, wonach der Arzt seiner Meinung nach verpflichtet gewesen sei. In diesem Zusammenhang ist $\mathrm{zu}$ bemerken, dass die Messung des Cuffdrucks eine medizinisch sehr sinnvolle Maßnah- 
me darstellt. Allein das Merkmal „sinnvoll“ qualifiziert eine Maßnahme jedoch noch nicht zum Standard.

Zur Prüfung des Sachverhalts wurden Lehrbücher der Anästhesiologie und die Gebrauchsinformation eines Tubusherstellers herangezogen $[4,7,8]$. Larsen [7] schrieb hierzu: „Der Füllungszustand des Cuffs soll... mittels Manometer geprüft werden. " [7]. Georgi et al. [4] führen aus: „Während der Langzeitintubation sollen die Cuff-Drücke... mit Manometern kontrolliert werden.“. Der Gebrauchsinformation des Herstellers des Tubus ist folgender Hinweis zu entnehmen: ,... sollte der Manschettendruck regelmäßig überwacht werden. “ [8]. Ist das Wort „soll“ ausreichend, um daraus ein „muss“, d. h. ein vom Anwender einzuhaltendes medizinisches Vorgehen, zu implizieren? Medizinische Lehrbücher werden von Ärzten und demzufolge juristischen Laien verfasst. Insofern ist die Bedeutung der medizinischen Umgangssprache zugrunde zu legen und nicht die Bedeutung des Wortes „soll“ aus verwaltungsrechtlicher Sicht. Der Gebrauch des Wortes „muss“ in den 3 zitierten Quellen [4, 7, 8] hätte dagegen die Messung des Cuffdrucks als Standard definiert.

\section{Gutachterkommissionen und Schlichtungsstellen}

In Deutschland können zur Klärung von Streitigkeiten zwischen Patienten und Ärzten, denen Schadensersatzforderungen von Patienten wegen vermuteter fehlerhafter ärztlicher Behandlung zugrunde liegen, neben ordentlichen Gerichten auch Gutachterkommissionen und Schlichtungsstellen (SST) angerufen werden. Antragsberechtigt sind Patienten, in Anspruch genommene Ärzte, Krankenhausträger und deren Versicherer. Das Verfahren wird schriftlich, freiwillig und für den Patienten kostenfrei durchgeführt. Die Kosten für einen Rechtsvertreter hat allerdings der Patient zu tragen [12]. Bei Verfahren vor SST hat der Patient - ebenso wie vor Zivilgerichten - zu beweisen, dass ein Behandlungsfehler den eingetretenen Gesundheitsschaden verursacht hat (Kausalität].

Die SST erfreuen sich einer hohen Akzeptanz bei Patienten, Ärzten und Juris-

Trauma Berufskrankh 2008 · 10[Suppl 2]:196-199 DOI 10.1007/s10039-008-1398-5

(c) Springer Medizin Verlag 2008

\section{W. Schaffartzik · J. Neu \\ Definition des ärztlichen Behandlungsfehlers}

\section{Zusammenfassung}

Die Unzufriedenheit des Patienten mit dem

Ergebnis der ärztlichen Behandlung stellt die wesentliche Ursache von Auseinandersetzungen zwischen Patient und Arzt dar. Nicht immer aber liegt dieser ein Behandlungsfehler zugrunde, andererseits führt nicht jeder Behandlungsfehler zu einem Gesundheitsschaden. Ein schuldhafter Behandlungsfehler liegt vor, wenn gegen anerkannte Regeln der Heilkunde verstoßen und die Sorgfalt, die von einem ordentlichen, pflichtgetreuen Arzt in der konkreten Situation erwartet werden kann, außer Acht gelassen wurden. Diese Einstufung bereitet Schwierigkeiten. Ist ein Gesundheitsschaden vom Patienten beweisbar auf einen Behandlungsfehler zurückzuführen, sind die Voraussetzungen zur Anerkennung von Schmerzensgeld gegeben. Zu Be-

\section{A definition of iatrogenic error}

\section{Abstract}

Patient dissatisfaction with the results of medical treatment is the main cause of conflict between patients and doctors. However, on the one hand this is not always due to a medical error, while on the other, a medical error does not always lead to damage to health. A culpable medical error has occurred when medical regulations have been contravened and the diligence expected from a proper, dutiful doctor in a particular situation has been neglected. Evaluating this is difficult. If a patient has suffered demonstrable damage to health and can prove that this was caused by a medical error, the conditions for compensation are fulfilled. The bur- weislasterleichterungen zugunsten des Patienten kommt es z. B. bei schweren Behandlungsfehlern, im Zusammenhang mit der Risikoaufklärung, bei Mängeln in der Befunderhebung und Dokumentation, in Fällen des Anscheinsbeweises und bei dem als vollbeherrschbar eingestuften Risiko. Bei Streitigkeiten spielen in Deutschland neben den Gerichten Gutachterkommissionen und Schlichtungsstellen eine wichtige Rolle. Um zukünftig Fehler zu vermeiden, kommt Fehlermeldesystemen eine wichtige Bedeutung zu.

\section{Schlüsselwörter}

Behandlungsfehler - Gesundheitsschaden . Beweislast · Schlichtungsstellen .

Fehlermeldesysteme den of proof is eased in favor of the patient in cases concerning e.g. serious medical errors, risk disclosure, incorrect evaluation and documentation of findings, circumstantial evidence and risk otherwise considered as fully controllable. In addition to the courts, advisory commissions and arbitration boards play an important role in conflicts in Germany. To avoid errors in the future, error alert systems are gaining in significance.

\section{Keywords}

Medical error - Damage to health · Burden of proof · Arbitration boards · Error alert systems 
Tab. 1 Fehler bezogen auf Sachentscheidungen im Jahr 2006

\section{Fehler} Häufigkeit [\%]

Behandlungsfehler, Aufklärungsmangel verneint Behandlungsfehler bejaht Behandlungsfehler, Aufklärungsmangel, Kausalität bejaht

Tab. 2 Verteilung der Patientenvorwürfe im Jahr 2006

\begin{tabular}{ll} 
Vorwurf & Häufigkeit [\%] \\
\hline Operative Therapie & 35,6 \\
\hline Bildgebende Verfahren & 5,5 \\
\hline Aufklärung, Risiko & 5,5 \\
\hline Konservative Therapie & 5,0 \\
\hline Medikamentöse Therapie & 4,0 \\
\hline
\end{tabular}

Tab. 3 Häufigste Diagnosen im Jahr 2006

\begin{tabular}{ll} 
Diagnose & Häufigkeit [\%] \\
\hline Koxarthrose & 3,3 \\
Gonarthrose & 2,5 \\
\hline $\begin{array}{l}\text { Unterschenkel-, } \\
\text { Sprunggelenkfraktur }\end{array}$ & 2,3 \\
Unterarmfraktur & 1,9 \\
\hline Mammakarzinom & 1,9 \\
\hline
\end{tabular}

ten. Die Qualität der Entscheidungen der SST, z. B. der Norddeutschen Schlichtungsstelle für Arzthaftpflichtfragen, ist u. a. daran zu erkennen, dass durch Schlichtungsverfahren in über 90\% der Fälle Gerichtsverfahren vermieden werden. Nur in $1,3 \%$ der Gerichtsverfahren, die einem Schlichtungsverfahren folgten, wurde gegen das Votum der Schlichtungsstelle entschieden [18].

\section{Zahl der Behandlungsfehler}

Spätestens seit der Veröffentlichung des Berichtes „To Err Is Human “ [6] im Jahr 1999 wird dem Auftreten von Behandlungsfehlern in der Medizin größte Beachtung geschenkt. Es ist nicht bekannt, wie viele Behandlungsfehler sich jährlich in Deutschland ereignen. Hansis u. Hart [5] gingen von etwa 40.00o Behandlungsfehlervorwürfen aus. Dabei könnten bis $\mathrm{zu}$ 12.00o Behandlungsfehler nachgewiesen worden sein [5]. Das Aktionsbündnis Patientensicherheit geht davon aus, dass bei $1 \%$ der Krankenhauspatienten Behandlungsfehler vorkommen [1].
Die Zahl der Behandlungsfehler ist derzeit in Deutschland nicht verlässlich festzustellen. Auch wiederholte Spekulationen über ihren Umfang mit sich überbietenden Zahlen ergeben kein gefestigtes Wissen. Die jüngste Initiative des Aktionsbündnisses Patientensicherheit e. V. widmet sich u. a. dem Thema, wie Ärzte aus Behandlungsfehlern lernen können [2].

Wie kann ein Behandlungsfehler erkannt werden? Sicher nicht nur an einem Gesundheitsschaden des Patienten; denn nicht jeder Behandlungsfehler führt zu einem Schaden. Ärztliche Aufgabe bleibt es, z. B. krankenhausintern Fehler bzw. fehlerträchtige Situationen zu erkennen, $\mathrm{zu}$ analysieren und $\mathrm{zu}$ vermeiden. Dies kann z. B. über das „Critical Incident Reporting System“ (CIRS, s. unten) geschehen. Darüber hinaus gestattet ein in der gesamten Bundesrepublik Deutschland von den SST verwendetes Fehlererfassungs- und -analysesystem (Medical Error Reporting System, kurz: MERS), Schadens- und Fehlerhäufungen zu detektieren [3]. MERS lässt nicht nur Fehler- und Schadenshäufungen erkennen, sondern gibt darüber hinaus auch Informationen zur Qualität der Gesundheitsschäden:

- Bagatellschaden vs. schwerer Schaden,

- passagerer Schaden vs. Dauerschaden bzw. Tod.

\section{Fehlermeldesysteme}

Gemeinsames und oberstes Ziel medizinischer Fehlermeldesysteme ist es, Schaden von den Patienten fernzuhalten. Dazu gehört, nicht nur durch Gesundheitsschäden der Patienten auf Fehler aufmerksam gemacht zu werden, sondern auch die Fehler zu erkennen, die (noch) nicht zu einem Schaden geführt haben. Deren Analyse muss wiederum dazu führen, dass sie vermieden werden können. Der nächste Schritt wäre es, keinerlei Fehler mehr entstehen zu lassen.

Im Folgenden werden 2 Fehlermeldesysteme dargestellt, die bei der Fehlerprävention an unterschiedlicher Stelle angreifen und sich in ihrer Analyse ergänzen.
Critical Incident Reporting System

Seine Einführung (s. unten) in Krankenhäusern und anderen Einrichtungen des Gesundheitswesens wurde vom Aktionsbündnis Patientensicherheit e. V. im Jahr 2007 empfohlen [1]. Ziel seiner Implementierung ist, zu einer Prävention und Reduktion von Behandlungsfehlern beizutragen.

Das System beruht auf einer freiwilligen, anonymen Meldung kritischer Ereignisse und Beinaheschäden. Es ist $\mathrm{u}$. E. insbesondere geeignet, Fehler vor Eintritt eines Gesundheitsschadens bei einem Patienten zu erkennen. Teil des Systems ist die Unterrichtung anderer Mitarbeiter, wodurch sie für die Situation sensibilisiert werden, damit sie den Fehler nicht begehen.

\section{Medical Error Reporting System}

Die „Ständige Konferenz der Gutachterkommissionen und Schlichtungsstellen“ hat entschieden, die Daten nach bundeseinheitlichen Parametern elektronisch im MERS zu erfassen. Dies gestattet eine Fehler- und Schadensanalyse der von den SST in der gesamten Bundesrepublik Deutschland bearbeiteten Fälle. Diese Bundesstatistik informiert neben Antrags- und Erledigungszahlen über die Zahl der festgestellten Behandlungsfehler sowie die Art, Häufung und Verteilung der Behandlungsfehler auf die medizinischen Fachgebiete [3].

MERS gestattet eine Analyse der Fehler- und Schadenshäufungen sowie der Fehlerursachen. Die Ergebnisse der Bundesstatistik können wissenschaftlich ausgewertet $[17,18,19]$ und für die Fortbildung und Qualitätssicherung im Zusammenhang mit der medizinischen Behandlung genutzt werden. Damit kann MERS zur Fehler- und Schadensvermeidung beitragen. So veröffentlichte die Schlichtungsstelle Hannover neben Manuskripten in wissenschaftlichen Journalen auch Falldarstellungen in den 9 regionalen Blättern der Landesärztekammern, die in der Schlichtungsstelle Hannover zusammengeschlossen sind $[13,14,15,16]$.

Ergebnisse MERS. Die Behandlungen, die Gesundheitsschäden hervorgeru- 
fen und die Patienten zu einer der Gutachterkommissionen oder Schlichtungsstellen geführt haben, sind in $68,6 \%$ im Krankenhaus und in $31,4 \%$ im niedergelassenen Bereich durchgeführt worden. Im Jahr 2006 wurden 10.255 Verfahren abgeschlossen und 7201 Sachentscheidungen getroffen. Dabei wurden in $28,3 \%$ Behandlungsfehler festgestellt (• Tab. 1). Von der Gesamtzahl der patientenseitig erhobenen Vorwürfe entfielen 35,6\% auf den chirurgischen Bereich (- Tab. 2), in welchem Schadenshäufungen zu erkennen sind. Hinsichtlich der 5 häufigsten Diagnosen (• Tab. 3) fällt auf, dass 4 dem Bereich Orthopädie und Unfallchirurgie zuzuordnen sind [3].

\section{Fazit}

Die Unzufriedenheit der Patienten mit dem Ergebnis der ärztlichen Behandlung stellt die wesentliche Ursache für Auseinandersetzungen zwischen Patient und Arzt dar. In Deutschland können zur Klärung von Streitigkeiten zwischen Patienten und Ärzten, denen Schadensersatzansprüche von Patienten wegen vermuteter fehlerhafter ärztlicher Behandlung zugrunde liegen, neben ordentlichen Gerichten auch ärztliche Gutachterkommissionen und Schlichtungsstellen (SST) angerufen werden. Gemeinsames und oberstes Ziel medizinischer Fehlermeldesysteme muss es sein, Schaden von den Patienten fernzuhalten. Dazu gehört es, nicht nur durch Gesundheitsschäden der Patienten auf Fehler aufmerksam gemacht zu werden, sondern auch die Fehler zu erkennen, die (noch) nicht zu einem Schaden geführt haben. Deren Analyse muss wiederum dazu führen, dass sie vermieden werden können. Dabei helfen Datenbanken weiter, wie sie der Bundesärztekammer mit dem „Medical Error Reporting System“ zur Verfügung stehen.

\section{Infobox 1: Internetlinks}

- Aktionsbündnis Patientensicherheit e.V.: http://www.aktionsbuendnis-patienten-

- Bundesärztekammer: http://www.bundesaerztekammer.de

- Schlichtungsstelle für Arzthaftpflichtfragen der Norddeutschen Ärztekammern: http://www.schlichtungsstelle.de

\section{Korrespondenzadresse}

\section{Prof. Dr. W. Schaffartzik}

Klinik für Anästhesiologie, Intensivmedizin und Schmerztherapie, Unfallkrankenhaus Berlin Warener Straße 7, 12683 Berlin

Walter.Schaffartzik@ukb.de

Interessenkonflikt. Keine Angaben sicherheit.de
13. Schaffartzik W (2002) Unzureichende Überwachung nach einer zahnärztlichen Operation in Narkose. Niedersachs Arztebl 5: 36

14. SchaffartzikW (2002) Postoperativer Kreislaufstillstand im Aufwachraum durch fehlerhaft durchgeführte Sauerstoffzufuhr. Niedersachs Arztebl I: 10 12

15. SchaffartzikW (2005) Misslungene tracheale Intubation. Niedersachs Arztebl 8: 36-37

16. SchaffartzikW (2007) Aspirationspneumonie durch Narkosefehler. Niedersachs Arztebl I: 24-25

17. SchaffartzikW, Neu J (2007) Schäden in der Anästhesie. Anästhesist 56: 444-448

18. Scheppokat KD (2005) Lehren aus den Erfahrungen einer Schlichtungsstelle für Arzthaftpflichtfragen. Ther Umschau 62: 185-190

19. Scheppokat KD, Held K (2002) Ergebnisse von 903 Schlichtungsverfahren in der Inneren Medizin. Dtsch Med Wochenschr 127: 253-259

20. Schlichtungsstelle für Arzthaftpflichtfragen der Norddeutschen Ärztekammern (2008) Verfahren/ Antrag. Schlichtungsstelle für Arzthaftpflichtfragen der Norddeutschen Ärztekammern, Hannover

\section{Literatur}

1. Aktionsbündnis Patientensicherheit e. V. (2007) Empfehlung zur Einführung von CIRS im Krankenhaus. Aktionsbündnis Patientensicherheit e.V., Bonn

2. Aktionsbündnis Patientensicherheit e. V. (2008) Aus Fehlern lernen. Aktionsbündnis Patientensicherheit e.V., Bonn

3. Bundesärztekammer (2007). Statistische Erhebung der Gutachterkommissionen und Schlichtungsstellen. Statistikjahr 2006. Bundesärztekammer, Berlin

4. Georgi R, Pothmann W, Krier C (2001) Sicherung der Atemwege. In: Kochs E, Krier C, Buzello W et al. (Hrsg) Anästhesiologie. Thieme, Stuttgart New York, S 330-369

5. Hansis ML, Hart D (2001) Medizinische Behandlungsfehler in Deutschland. In: Robert Koch-Institut (Hrsg) Gesundheitsberichterstattung des Bundes. Fieck, Berlin, S 4-13

6. Kohn LT, Corrigan JM, Donaldson MS (eds) (1999) To err is human: building a safer health system. Committee on Quality of Health Care in America. National Academy Press, Washington DC

7. Larsen R (2006) Anästhesiologie 21. Endotracheale Intubation und Larynxmaske, 8. Aufl. Urban \& Fischer, München, S 497-554

8. Mallinckrodt (2002) Packungsbeilage Tubus. CE 0050, Revision 2, MAP0001. Mallinckrodt, Hazelwood

9. Neu J (2001) Arzthaftungsrecht 11. Ärztlicher Standard. In: Neu J, Petersen D, Schellmann WD (Hrsg) Arzthaftung/Arztfehler. Steinkopff, Darmstadt, $S$ 427-428

10. Neu J (2001) Arzthaftungsrecht 12. Ärztliche Sorgfalt, Fahrlässigkeit, Behandlungsfehler. In: Neu J, Petersen D, Schellmann WD (Hrsg) Arzthaftung/ Arztfehler. Steinkopff, Darmstadt, S 429-431

11. Neu J (2001) Arzthaftungsrecht 13. Schwerer (grober) Behandlungsfehler. In: Neu J, Petersen D, Schellmann WD (Hrsg) Arzthaftung/Arztfehler. Steinkopff, Darmstadt, S 432-434

12. Püschmann H, Neu J (2005) Das Schlichtungsverfahren der Norddeutschen Ärztekammern. SchlHA 12: $406-408$ 